\title{
Dynamics of planar rocking-blocks with Coulomb friction and unilateral constraints: comparisons between experimental and numerical data
}

\author{
Hongjian Zhang • Bernard Brogliato • Caishan Liu
}

\begin{abstract}
This paper concerns the dynamics of planar rocking blocks, which are mechanical systems subject to two unilateral constraints with friction. A recently introduced multiple impact law that incorporates Coulomb friction is validated through comparisons between numerical simulations and experimental data obtained elsewhere by other authors. They concern the free-rocking motion with no base excitation, and motions with various base excitations for the study of the onset of rocking and of the overturning phenomenon. The comparisons made for free-rocking and for the onset of rocking demonstrate that the proposed impact model allows one to correctly predict the block motions. Especially the free-rocking experiments can be used to fit the impact law parameters (restitution and friction coefficients, block width). The free-rocking fitted parameters are then used in the excited-base cases.
\end{abstract}

Keywords Rocking block · Multiple impacts · Coulomb friction · Free-rocking · Onset of rocking · Overturning

\section{Introduction}

Modeling the dynamics of a rigid block hitting a rigid ground has attracted the attention of scientists in the field of earthquake engineering for a long time; see, e.g., [5, 22, 32, 42,

H. Zhang · C. Liu

State Key Laboratory for Turbulence and Complex Systems, College of Engineering, Peking University, Beijing 100871, People's Republic of China

H. Zhang

e-mail: zhanghj@pku.edu.cn

C. Liu

e-mail: liucs@pku.edu.cn

B. Brogliato

INRIA, BIPOP project-team, ZIRST Montbonnot, 655 avenue de l'Europe, 38334 Saint Ismier, France e-mail: bernard.brogliato@inria.fr 
$46,53,54,59,60]$ to cite a few. In parallel, the field of impact dynamics has witnessed an intense activity in the past 25 years; see, e.g., [8-10, 15, 18-20, 29, 31, 38, 48, 52, 66] and references therein. It happens that the problem of modeling impacts with friction is a tough issue, especially when there are several simultaneous contact points (multiple impacts with friction). Typically, the so-called rocking block problem involves double-impacts with friction, when one assumes that the base contacts the ground at two points only. Together with chains of balls, the rocking block is an apparently a simple multibody system (the block and the ground); however, it involves multiple impacts with friction and its modeling is consequently not simple at all.

In [7], experimental tests were led and compared to the model with Housner angular restitution $r$ [22] calculated from the conservation of angular momentum before and after the shock. It was found that the analytical value of $r=-\frac{2 l^{2}-L^{2}}{2 L^{2}+2 l^{2}}$, where $l$ and $L$ are the block height and width, respectively, did not match with the experimental one. Similar conclusions were drawn in [45] who found a rather big discrepancy between the analytical and the experimental values of $r$. Usually the experimentally measured values for $r$ are larger than the theoretically predicted ones, and many authors simply fit $r$ with the data without questioning the model [7, 32, 45, 50, 54]. Recently experimental tests on concrete blocks have shown [16] that the ratio between the measured $r$ and the above one may be smaller or larger than one, despite the fact that it is generally found to be smaller (see Table II in [16]), contradicting the older conclusions. These authors also showed the inability of the kinematic angular restitution law to predict the free-rocking motion (see Fig. 18 in [16]). Lispcombe et al. [32] calculated $r$ by introducing the kinematic restitution and adding constraints for no slipping or unidirectional slip. The fact that a block rotating around one corner and impacting at the other corner may rebound at both corners (and thus become airborne) is studied in [32]. Most importantly, these authors also proved experimentally the existence of sequences of impacts at the corner point around which the block rotates during a rocking motion. The number of such impacts, and the duration of the rebound phase, depend on the block aspect ratio $\frac{l}{L}$. Yilmaz et al. [59] used a generalization of Routh's approach and so-called impulse correlation ratios, without friction. They found good agreements between their simulations and their experiments. Pena and Prieto et al. [42, 43, 46] performed many experiments and also proposed a new model for rocking. It is noteworthy that in their experiments that Pena et al. [42] found better matching between the above value of $r$ and the experimental values, which differ by much smaller percentage than in [7, 45]. Some authors like Palmeri et al. [41] introduced compliance at the contacts and frictional effects in order to cope with such complex dynamics. In [5], the nonsmooth mechanics framework is adopted and friction with a nonconstant sliding coefficient is used. Taniguchi [53] uses the Housner angular velocity restitution coefficient, and Coulomb's law during nonimpacting phases of motion. He points out that perfect rocking seldom occurs, whereas stick/slip phases may be the common behavior. In [11, Sect. 5], it is shown that if rebounds are allowed at the corners, $r$ may lack of physical meaning as it cannot be uniquely chosen given a unique energetical behavior. It is also shown that Housner coefficient is a lower bound in the space of admissible coefficients, corroborating observations in [17]; see [11, Table 1]. The conclusions to be drawn from all these works are that experimental results are not always easy to interpret, and kinematic restitution rules applied to planar models are too simplistic to correctly model the motion of a block rebounding on a ground, which may consist of complex stick/slip/impact/rebound phases. Recently, three-dimensional models have been developed [67], thus relaxing the assumption that the system is confined in a $2 \mathrm{D}$ motion.

The objective of this work is to demonstrate that the multiple impact model introduced in $[33-36,65]$ and named in the sequel the LZB model, can be useful for the study of the 
rocking block dynamics (free-rocking when the base is fixed, and with various base excitations). This is shown through detailed comparisons between numerical results obtained with the LZB model, and experimental results obtained in Pena et al. [42, 43]. Those comparisons concern free-rocking (fixed base) and the onset of rocking (with horizontal base excitation). The conclusions are quite positive and confirm the results obtained in $[34,36,39,65]$ where detailed comparisons with experimental data for Newton's cradle [36], bouncing dimer [65], column of beads [34], and tapered chains [39] are made: The LZB model does encapsulate the main dynamical effects of multiple impacts with Coulomb friction. It is noteworthy that our model seems to be able to predict the motion for systems ranging from few grams (the dimer [65]) to several hundreds of kilograms (the rocking blocks in [42, 43]). Most importantly, it is shown that the free-rocking experiments can be used to fit the parameters (restitution coefficients, friction coefficients, block width), which are used to predict more complex motions with base excitation. The paper is organized as follows: In Sect. 2, the planar block dynamics and the multiple impact model are introduced. Section 3 is dedicated to the comparisons between the experimental data in $[42,43]$ and numerical results, for freerocking motion. Sections 4, 5, and 6 deal with the case of base excitation: Rocking motion, the onset of rocking, and the overturn phenomena are studied. Conclusions end the paper in Sect. 7 .

\section{The block dynamics and the impact law}

In this section, we introduce the dynamical model of the two-body block/anvil system, which will be used to perform the numerical simulations.

\subsection{The block dynamics outside the collisions}

Let us consider the block as a three degree-of-freedom planar homogeneous solid, with generalized coordinates $q^{T}=(x, y, \theta)$, where $x$ and $y$ are the horizontal and vertical positions of the center of gravity, $\theta$ is the angular position; see Fig. 1 . The base (the anvil) is a one

Fig. 1 The planar block with horizontally moving base

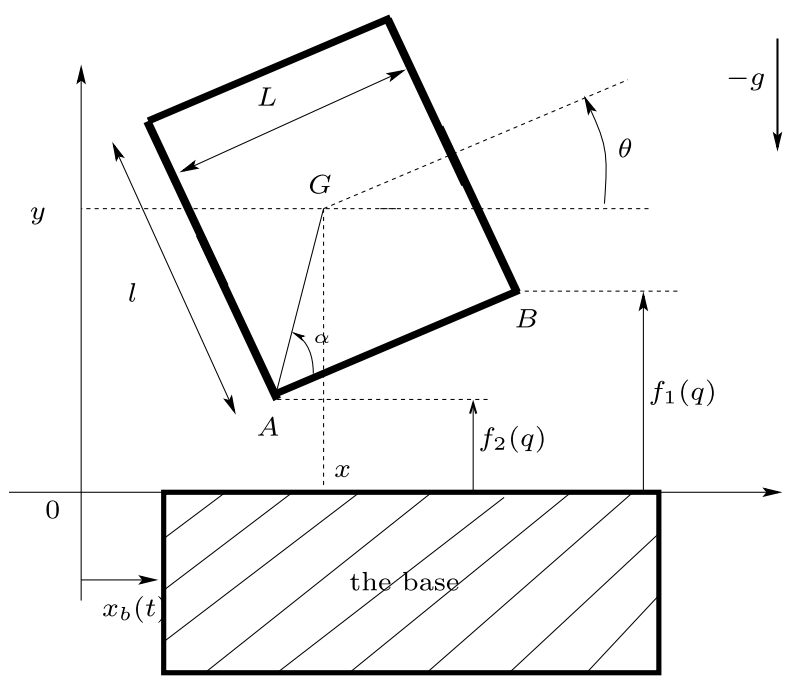


degree-of-freedom system with mass $m_{b}$, moving in translation along the $x$-axis. Its position is denoted as $x_{b}$. Let us make the following assumptions.

Assumption 1 The block/anvil contact can be represented by two points $A$ and $B$ at the corners.

\section{Assumption 2 The dynamical effects of the block on the anvil are neglected.}

Assumption 1 is common in the rocking block literature. Its validity will be discussed in this paper. Assumption 2 is motivated by the fact that we shall use experimental results from [42, 43]. Actually, it is indicated in [43] that the uniaxial shaking table used in their experiments has a total mass $m_{b}=6$ tons, while the blocks have masses ranging from 120 to $500 \mathrm{~kg}$. Hence, $\frac{m}{m_{b}} \in[0.02,0.08]$. In fact, assumption 2 means that one neglects all vertical motions (velocity jumps at impacts, vibrational effects) in the mechanical structure which makes the base.

Following [10, Chap. 6], we infer that the block, when $y \leq \frac{\sqrt{l^{2}+L^{2}}}{2}$, is subject to two unilateral constraints:

$$
\left\{\begin{array}{l}
f_{1}(q)=y-\frac{l}{2} \cos (\theta)+\frac{L}{2} \sin (\theta) \geq 0, \\
f_{2}(q)=y-\frac{l}{2} \cos (\theta)-\frac{L}{2} \sin (\theta) \geq 0,
\end{array}\right.
$$

where $f_{1}(q) \geq 0$ expresses that point $B$ cannot penetrate into the base, while $f_{2}(q) \geq 0$ expresses the same for point $A$. Given the above assumptions, the dynamics of the block subject to (1) and Coulomb friction is given by:

$$
\left\{\begin{array}{l}
\begin{array}{l}
m \ddot{x}(t)=\lambda_{t, 1}(t)+\lambda_{t, 2}(t), \\
m \ddot{y}(t)=\lambda_{n, 1}(t)+\lambda_{n, 2}(t)-m g,
\end{array} \\
\quad \begin{array}{rl}
I_{G} \ddot{\theta}(t)= & \lambda_{n, 1}(t)\left(\frac{l}{2} \sin (\theta(t))+\frac{L}{2} \cos (\theta(t))\right)+\lambda_{n, 2}(t)\left(\frac{l}{2} \sin (\theta(t))-\frac{L}{2} \cos (\theta(t))\right) \\
& \quad+\left(\frac{l}{2} \cos (\theta(t))-\frac{L}{2} \sin (\theta(t))\right) \lambda_{t, 1}+\left(\frac{l}{2} \cos (\theta(t))+\frac{L}{2} \sin (\theta(t))\right) \lambda_{t, 2},
\end{array} \\
\begin{array}{rl}
0 \leq \lambda_{n}(t) \perp f(q(t)) \geq 0, \\
\lambda_{t, i}(t) \in-\mu_{i} \lambda_{n, i}(t) \operatorname{sgn}\left(v_{t, i}(t)-v_{b}(t)\right), \quad i=1,2,
\end{array}
\end{array}\right.
$$

where $v_{b}(t)=\dot{x}_{b}(t)$ is the base horizontal velocity, $\mu_{i}>0$ is the friction coefficient at contact $i$, and $v_{t, i}$ is the tangential velocity at the point $i$, i.e., $v_{t, 1}=\dot{x}+\left(\frac{l}{2} \cos (\theta)-\frac{L}{2} \sin (\theta)\right) \dot{\theta}$ at $B$ and $v_{t, 2}=\dot{x}+\left(\frac{l}{2} \cos (\theta)+\frac{L}{2} \sin (\theta)\right) \dot{\theta}$ at $A$ (from which $v_{t, 1}=v_{t, 2}$ when $\theta=0$ ). Notice that if the contact point $i$ detaches then the complementarity conditions imply that $\lambda_{n, i}=0$ so $\lambda_{t, i}=0$. The complementarity conditions are componentwise, $f(q)^{T}=\left(f_{1}(q), f_{2}(q)\right)$, $\lambda_{n}^{T}=\left(\lambda_{n, 1}, \lambda_{n, 2}\right)$. For a block with $G$ at the geometric center, one has $I_{G}=\frac{m}{12}\left(l^{2}+L^{2}\right)$. In (2), we have not yet considered the impacts with the ground, but only those phases of motion where the contact force is a bounded function of time. It is possible to rewrite compactly the smooth part of the dynamics in (2) as

$$
M \ddot{q}(t)=W_{n}(q(t)) \lambda_{n}(t)+W_{t}(q(t)) \lambda_{t}(t)-\mathbf{g}
$$

with $\mathbf{g}=(0 m g 0)^{T}, M=\operatorname{diag}\left(m, m, I_{G}\right) ; W_{n}(q)$ and $W_{t}(q)$ are easily identified from (2). One has $v_{n}=W_{n}^{T}(q) \dot{q}, v_{t}=W_{t}^{T}(q) \dot{q}$, where $v_{n}=\left(v_{n, 1} v_{n, 2}\right)^{T}, v_{t}=\left(v_{t, 1} v_{t, 2}\right)^{T}$ denote the local velocities of the contact points [2]. From (1) and (2), the complementarity problem that 
allows one to calculate the contact forces during the smooth phases of motion (i.e., outside impacts) is given by

$$
0 \leq \lambda_{n}(t) \perp A(\theta) \lambda_{n}(t)+W_{n}(q) M^{-1} W_{t}(q) \lambda_{t}(t)+B(\theta, \dot{\theta}) \geq 0,
$$

with

$$
A(\theta)=\left(\begin{array}{cc}
\frac{1}{m}+\frac{1}{4 I_{G}}(l \sin (\theta)+L \cos (\theta))^{2} & \frac{1}{m}+\frac{1}{4 I_{G}}\left(l^{2} \sin ^{2}(\theta)-L^{2} \cos ^{2}(\theta)\right) \\
\frac{1}{m}+\frac{1}{4 I_{G}}\left(l^{2} \sin ^{2}(\theta)-L^{2} \cos ^{2}(\theta)\right) & \frac{1}{m}+\frac{1}{4 I_{G}}(l \sin (\theta)-L \cos (\theta))^{2}
\end{array}\right),
$$

and

$$
B(\theta, \dot{\theta})=\left(\begin{array}{l}
-g+\frac{1}{2} \dot{\theta}^{2}(l \cos (\theta)-L \sin (\theta)) \\
-g+\frac{1}{2} \dot{\theta}^{2}(l \cos (\theta)+L \sin (\theta))
\end{array}\right) .
$$

The matrix $A(\theta)=W_{n}^{T}(q) M^{-1} W_{n}(q)$ is the so-called Delassus' matrix of the system (1)-(2). The details on how to analyze and solve such problems is outside the scope of this paper; see [2,35]. Let us just mention that, in general, friction may create inconsistencies and indeterminacies [10, Sect. 5.5] yielding Painlevé paradoxes. Such issues are met only for unrealistic values of friction in planar blocks [30, Appendix A].

\subsection{The impact dynamics}

The impact model proposed in [33-36, 65] is summarized in this section. It will be named the LZB impact model in the following (from the authors' names). The contact stiffnesses are denoted as $k_{i}$, the elasticity potential energy at contact $i$ is $E_{i}, \eta$ is the elasticity coefficient. The matrix $W_{n}(q)$ is the Jacobian between the generalized velocities $\dot{q}$ and the contact points normal relative velocities, i.e., $W_{n}^{T}(q)=\frac{\partial f}{\partial q}(q)=\nabla f^{T}(q) \in \mathbb{R}^{2 \times 3}$, whereas $W_{n}(q) \lambda_{n}$ represents the generalized contact force associated with the generalized coordinates $q$; see (2) and (3). The scalar $P_{n, i}$ denotes the normal component of the interaction force impulse at contact point $i, P_{n}=\left(P_{n, 1}, P_{n, 2}\right)^{T}$ is the vector of normal impulses. An important assumption in this model, that is an extension of the Darboux-Keller approach [10], is that positions $q$ are constant during the impact process. Thus, $W_{n}(q)$ is supposed to be constant during an impact and is simply denoted as $W_{n}$. In the frictionless case, the LZB impact dynamics is summarized as follows:

- Contact parameters: $\gamma_{j i}=\frac{k_{j}}{k_{i}}$ (normal contact stiffnesses ratios), $e_{n, j}$ (energetic restitution coefficients), $1 \leq i \leq 2,1 \leq j \leq 2, \eta$ (=1 for linear elasticity, $=\frac{3}{2}$ for Hertz contact, or other values).

- Dynamical equation:

$$
M \frac{d \dot{q}}{d P_{n, i}}=W_{n} \frac{d P_{n}}{d P_{n, i}} \quad \text { if } E_{j i}\left(P_{n, j}, P_{n, i}\right) \leq 1 \quad \text { for } j \neq i
$$

with the distributing law: ${ }^{1}$

$$
\frac{d P_{n, j}}{d P_{n, i}}=\gamma_{j i}^{\frac{1}{\eta+1}}\left(E_{j i}\left(P_{n, j}, P_{n, i}\right)\right)^{\frac{\eta}{\eta+1}}
$$

\footnotetext{
${ }^{1}$ The power of the potential energies ratio $E_{j i}\left(P_{n, j}, P_{n, i}\right)$ is inverted in [33, 34].
} 
Fig. 2 Bistiffness contact compliant model

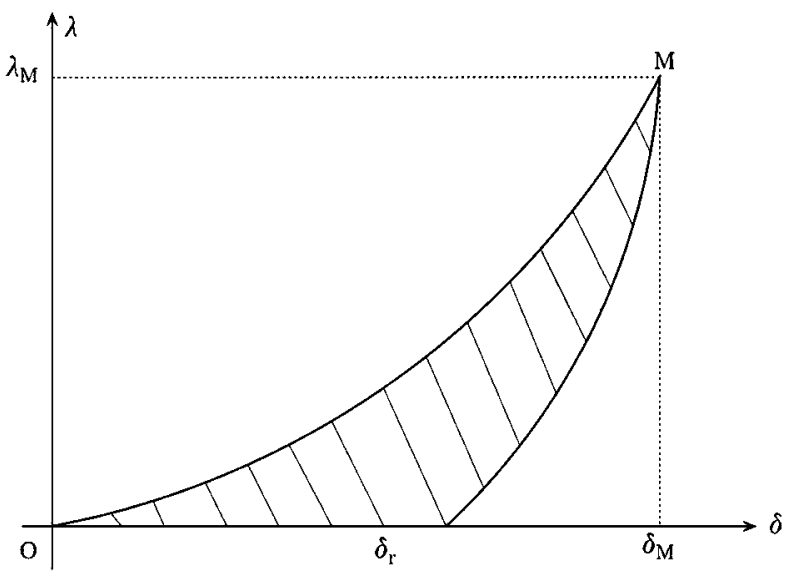

and the potential energies ratios:

$$
E_{j i}\left(P_{n, j}, P_{n, i}\right)=\frac{E_{j}\left(P_{n, j}\right)}{E_{i}\left(P_{n, i}\right)}, \quad 1 \leq i \leq 2, \quad 1 \leq j \leq 2,
$$

where

$$
E_{j}\left(P_{n, j}\right)=\int_{0}^{P_{n, j}(t)} \mathbf{w}_{j}^{T} \dot{q} d P_{n, j}
$$

The vectors $\mathbf{w}_{j} \triangleq \nabla f_{j}$ are the columns of the Jacobian matrix $W_{n}$. The impulse $P_{n, i}$ at contact $i$ is the so-called principal impulse that is chosen as the new time-variable in the impact model. It may change during the impact process; see [33] for details. The compliance effect is added into LZB model by using a contact model such as the monostiffness or bistiffness compliance models. We limit ourselves to the bistiffness as presented in [21, pp. $92,107]$, which aims to incorporate elastic effects together with plastic, damage and other irreversible effects. It has been experimentally verified for sphere/sphere impacts in several papers; see, e.g., $[6,12,13,51,56,58]$. As illustrated in Fig. 2, for the bistiffness compliance model, the force-indentation relationship at a given contact point $j$ for the compression phase is expressed as

$$
\lambda_{c, j}=k_{j}\left(\delta_{j}\right)^{\eta}
$$

It is different from that for the expansion phase expressed as

$$
\lambda_{e, j}=\lambda_{M, j}\left(\frac{\delta_{j}-\delta_{r, j}}{\delta_{M, j}-\delta_{r, j}}\right)^{\eta},
$$

where $\delta_{r, j}$ is the plastic deformation, and $\lambda_{M, j}$ and $\delta_{M, j}$ are respectively the maximum values of the normal contact force and of the normal deformation at the contact point $j$ at the end of the compression phase (when $\dot{\delta}_{j}=0$ ). The maximum compression is attained at the point $M$ in Fig. 2, and $\delta_{r}$ is the residual indentation. The time $t_{c}$ of maximal compression at the contact $j$ is calculated from $\dot{\delta}_{j}\left(t_{c}\right)=0$ where $\delta_{j}$ is the relative normal displacement at contact $j\left(\dot{\delta}_{j}=\mathbf{w}_{j}^{T} \dot{q}=\nabla f_{j}^{T} \dot{q}\right)$. For a single impact at contact $j$, the termination time $t_{f}$ is 
Fig. 3 Contact point experiencing multiple compression and expansion phases

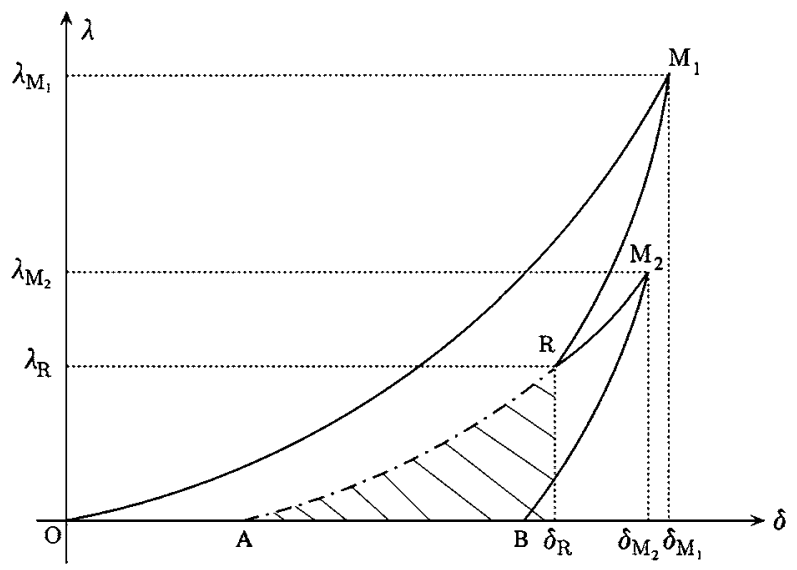

calculated from the energy constraint $W_{r, j}=-e_{n, j}^{2} W_{c, j}$, where the works during compression and expansion phases are given by, respectively,

$$
W_{c, j}=\int_{0}^{P_{n, j}\left(t_{c}\right)} \mathbf{w}_{j}^{T} \dot{q} d P_{n, j}, \quad W_{r, j}=\int_{P_{n, j}\left(t_{c}\right)}^{P_{n, j}\left(t_{f}\right)} \mathbf{w}_{j}^{T} \dot{q} d P_{n, j} .
$$

Thus, the residual indentation $\delta_{r, j}$ at contact $j$ is a function of $e_{n, j}$, and $\delta_{r, j}=$ $\delta_{M, j}\left(1-e_{n, j}^{2}\right)$ for constant $e_{n, j}$; see [33, Eq. (3.4)]. When a contact experiences multiple cycles of compression and expansion phases, secondary cycles can be treated as the case of single cycles with an initial potential energy. The initial potential energy of each secondary cycle is the one computed for the preceding cycle at the point where the secondary cycle under consideration starts. To illustrate this, we consider a contact experiencing two compression and expansion phases shown in Fig. 3. The secondary cycle starts at the point $R$, follows the compression curve $\widehat{R M_{2}}$ and then the expansion curve $\widehat{M_{2} B}$, and finally stops at the point $B$ where the potential energy is entirely released or dissipated. This secondary cycle is treated as a single cycle whose initial potential energy $E_{o, j}$ is the potential energy at the point $R$ computed for the cycle $\widehat{O M_{1} R}$. As proved in [33], the distributing law in (8) remains valid for multiple compression/expansion phases, i.e., more than two compression/expansion cycles at the same contact point.

One has $e_{n, j} \in[0,1]$, which means that the work performed by the normal force during the expansion cannot exceed the energy "injected" in the system during the compression (when several compression/expansion phases exist at contact $j$ the energetical constraint is applied to the whole contact process at this point). In view of (2), in the frictionless case, the impact dynamics have reduced dimension because $\frac{d \dot{x}}{d P_{n, i}}=0$, hence the coordinate $x$ plays no role in the analysis; see (2) with $\lambda_{t, 1}(t)+\lambda_{t, 2}(t)=0$. Coulomb friction can be easily added in the impact model, at the force (or infinitesimal impulse) level [35, 65]. In such a case, the right-hand side of (7) has to be modified accordingly with the insertion of the tangential force components; see (2) and (3):

$$
M \frac{d \dot{q}}{d P_{n, i}}=W_{n} \frac{d P_{n}}{d P_{n, i}}+W_{t} \frac{d P_{t}}{d P_{n, i}} \quad \text { if } E_{j i}\left(P_{n, j}, P_{n, i}\right) \leq 1 \quad \text { for } j \neq i .
$$

Remark 1 In this paper, the basic Coulomb's law is enhanced to incorporate static $\mu_{s}$ and dynamic $\mu$ friction coefficients; see Fig. 4. Also, it will be assumed in all the following simu- 
Fig. 4 The friction model, $i=1,2$

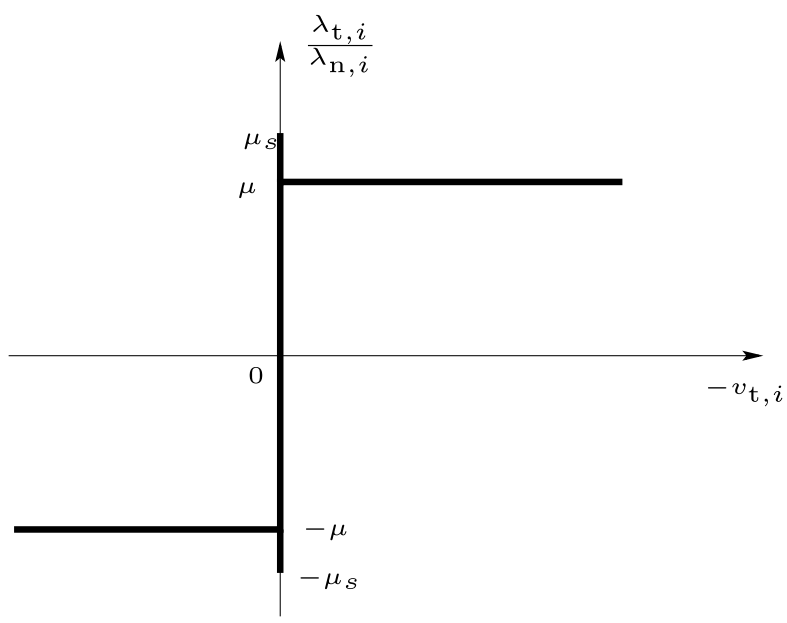

lated examples that $\mu_{1}=\mu_{2}=\mu$ and $\mu_{s, 1}=\mu_{s, 2}=\mu_{s}$, in accordance with the experimental data taken in $[42,43]$.

This impact model is therefore a rigid body model that incorporates some flexibility effects through the distributing law, with one restitution coefficient and one (or two) friction coefficient per contact. In this work, we shall use the same static and dynamic coefficients of friction for the two contact points. More details on the implementation may be found in $[35,36,40]$. For the dynamics outside the impacts, the complementarity problem in (4) is used to integrate the system, and either an explicit Euler or Runge-Kutta algorithms are implemented. It is noteworthy that the numerical scheme that is employed next is of the event driven type [2], and that all the stick/slip and contact/detachment conditions are carefully taken care of (taking care, in particular, of the multivalued feature of the friction law at zero tangential velocity). To summarize, we use an event-driven method with the complementarity model (2) outside impacts and the above LZB impact dynamics when an impact is detected. In the rest of the paper, it will be assumed that the normal restitution is identical for the two contact points, denoted as $e_{n}^{*}$. The impact LZB model applied to the block/anvil system therefore has a total of 5 parameters including friction: $e_{n}, \eta, \gamma_{12}=\frac{k_{1}}{k_{2}}, \mu, \mu_{s}$.

Remark 2 An interesting feature of the LZB impact law is that it allows for stick/slip transitions during the impacts, as shown in [63, 64]. This means that the relative tangential velocity can reverse its sign during the collision without implying energetical incoherencies, as it is the case with kinematic restitution laws [11, 15]. Tangential velocity reversals do occur in the planar block; see Fig. 21(b) in [63]. Notice that the complementarity modeling in (2) has been used before for block/ground systems in [5, 44, 49, 62]. Finally, it is noteworthy that rocking motions cannot be described by kinematic normal restitution laws coupled to Coulomb friction [11]. This means that kinematic restitution laws are unable to model dry friction effects and rocking motions together. Other models have been introduced $[4,24]$, which allow for different modes (airborne block, stick/slip transitions). Andreaus and Casini [4] use a compliant contact/impact model, whereas [24] uses a rigid body model but no complementarity relations, and the friction during impacts is simply introduced with constant kinematic tangential restitution coefficients. 


\section{Free-rocking experiments}

In this section, it is proved that the LZB model can be fitted to provide correct predictions of free-rocking motions, with fixed base. Free-rocking corresponds to the block initialized with a nonzero angle $\theta(0)$, one corner in contact, and zero initial velocity $\dot{\theta}$. Experimental data led on blue granite stone blocks are reported in [42, 43]. In the following, we provide detailed comparisons between the numerical simulations obtained with the LZB model and the experimental data of $[42,43] .^{2}$ The tests concern four specimens of blocks. The masses of the blocks are estimated from their dimensions and density, and are given by $503 \mathrm{~kg}$, $228 \mathrm{~kg}, 120 \mathrm{~kg}, 245 \mathrm{~kg}$, for specimens 1, 2, 3, 4, respectively. It has been shown in [43] that specimen 3 is prone to significant three-dimensional effects like torsional and vibrational effects. Due to this, the planar model could not satisfactorily reproduce the experimental $\theta(t)$ response. Similar difficulties were found in [42] to calibrate a DEM model on specimen 3 data (see Table III in [42]). The results for specimen 3 are thus not presented in this paper. Specimens 1, 2, and 4 experimentally showed no or negligible three-dimensional effects [43, Sect. 8.1].

\subsection{The $\theta(t)$ response}

The results are depicted in Figs. 5(a), (b), and (c). The fitted restitution coefficients are $e_{n}=0.999$ for specimen 2 in Fig. $5(\mathrm{~b})$, and $e_{n}=0.97$ before $2.83 \mathrm{~s}, e_{n}=0.88$ after $2.83 \mathrm{~s}$ for specimen 1 in Fig. 5(a). For specimen 4, the fitted values are $e_{n}=0.99$ before $0.85 \mathrm{~s}$, $e_{n}=0.84$ after $0.85 \mathrm{~s}$ (see comments (i) and (ii) in Sect. 3.2). The fitted dimensions are $\frac{l}{L}=\frac{1}{0.23} \approx 4.35$ for specimen 1 and $\frac{l}{L}=\frac{1}{0.155}=6.45$ for specimen $2, \frac{l}{L}=\frac{0.50}{0.115}=4.34$ for specimen 4. The friction coefficients are chosen as $\mu=0.3$ and $\mu_{s}=0.577$ as in [43]. The SRM curves correspond to the response calculated with the Housner angular restitution coefficient (as recalled in Sect. 1).

Uncertainties in $e_{n}$ and $L$ It happens that the rocking motion is highly sensitive to parameters variations, which renders the calibration of the parameters a delicate process. This is illustrated in Figs. 5(b) and 6; see also Fig. 41 in [63], where it is shown that very small variations on $e_{n}$ and $L$ produce large variations on the $\theta(t)$ response. The uncertainty in the width $L$ affects mainly the pseudo frequency of the oscillations and has little effect on the magnitudes. This is in contrast with uncertainties on $e_{n}$, which affect both the frequency and the magnitude. The various widths, which enter the study are recapitulated in Table 1 . The geometric widths are those measured on the blocks. The experimental widths are obtained from an estimation process using the Housner model; see Sect. 4.1 in [42]. The other two sets of widths are obtained by fitting the parameters. The larger width discrepancy for specimen 4 comes from large cuts at the corners on this block. The choices for smaller widths in simulation are in agreement with the conclusion in [47], where what we call the width in simulation may be called the effective width following [47]. In short, due to the contact line geometry imperfections, the equivalent width of the model with two contact points must be smaller than the geometrical width of the real block. See also Sect. 3.2(ii) below.

\footnotetext{
${ }^{2}$ All the experimental data used for the comparisons with numerical data presented in this paper have been made available to us by Dr. F. Pena from the Instituto di Ingenieria, UNAM, Mexico. They correspond to the data in the various figures and tables in [42,43]. They can be consulted in Tables 3-8 in [63].
} 


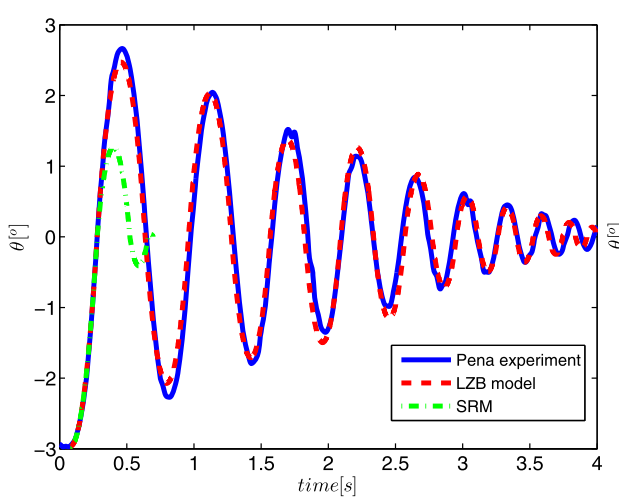

(a) Specimen 1. $e_{\mathrm{n}}=0.97$ and $0.88, l=1 \mathrm{~m}$, $L=0.23 \mathrm{~m}$.

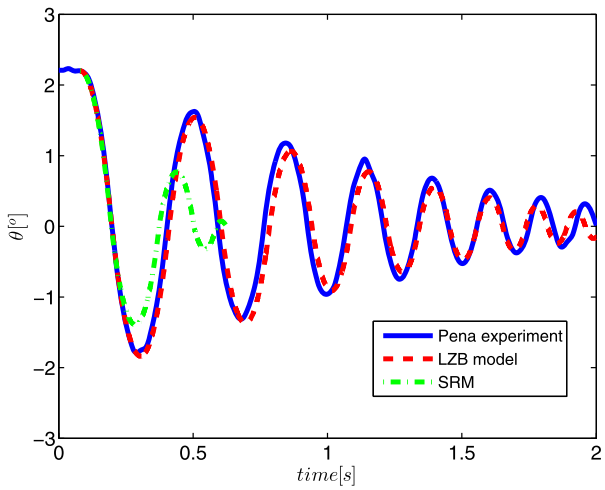

(c) Specimen $4, e_{\mathrm{n}, i}=0.99$, and $0.87, l=$ $0.50 \mathrm{~m}, L=0.115 \mathrm{~m}$.

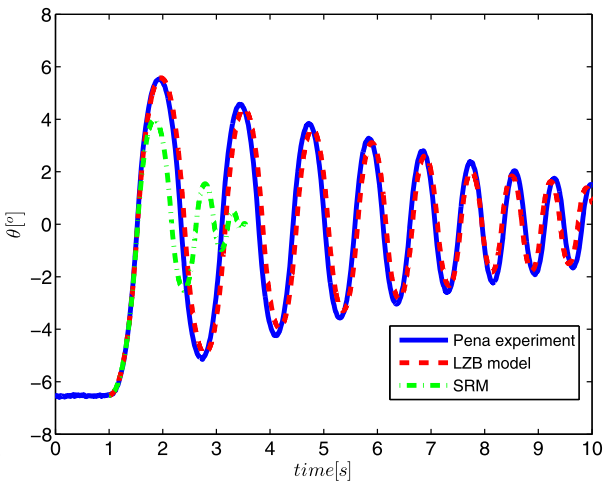

(b) Specimen 2. $e_{\mathrm{n}}=0.999, l=1 \mathrm{~m}, L=$ $0.155 \mathrm{~m}$.

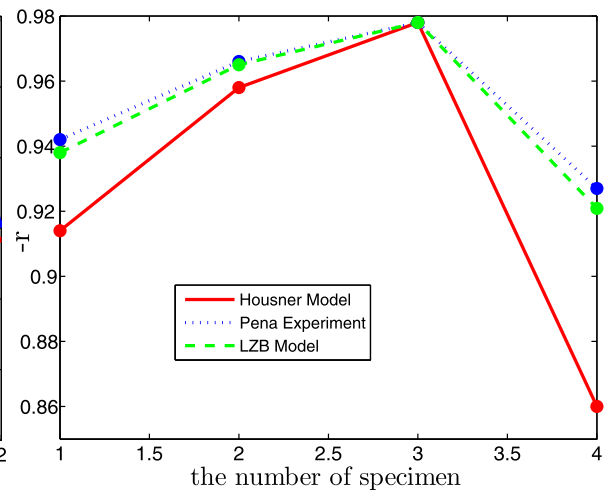

(d) The angular restitution coefficient $r$ (fitted parameters).

Fig. 5 Numerical $v s$. experimental values of $\theta(t)$ and $r$

Table 1 The various widths

\begin{tabular}{llll}
\hline & 1 & 2 & 4 \\
\hline Geometric width (m) & 0.24 & 0.16 & 0.15 \\
Experimental width (m) & 0.2394 & 0.1645 & 0.1255 \\
Width in simulations of [43] (m) & 0.2468 & 0.1696 & 0.1464 \\
Width in LZB model (m) & 0.23 & 0.155 & 0.115 \\
\hline
\end{tabular}

The elasticity coefficient $\eta$ and normal stiffnesses The elasticity coefficient enters the LZB impact model through the co-called distributing law and the potential energies ratios $E_{j i}\left(P_{j}, P_{i}\right)$. In all the simulations, we have made the choice $\eta=\frac{3}{2}$ (Hertz contact). It happens that the potential energies ratios are small enough so that changing $\eta$ from 1 to $\frac{3}{2}$ does not much influence the results. Also we always chose stiffnesses ratios equal to one for obvious physical reasons (the materials characteristics are the same at both contact points). Notice that only the stiffnesses ratios play a role in the impact dynamics, not their absolute values (this fact holds true for other types of multibody systems with several contact points; see [10, Claim 6.3]). 


\subsection{Comments}

(i) Some fitted values for $e_{n}$ (i.e., 0.999 or 0.99 ) are very close to one, showing very small amounts of kinetic energy loss at each impact. Experimental works on single granite/granite impacts without friction report values of the restitution coefficient in [0.873, 0.897], and varying nonmonotonously with the height drop [23, Fig. 6]. It is also known from many experimental results that the restitution coefficient usually tends to 1 when the initial relative velocity tends to zero; see, e.g., [51]. In the presented results, the normal relative velocity is very small (about $0.02 \mathrm{~m} / \mathrm{s}$ ). The values for the angular restitution coefficient $r$ calculated in Table II in [42] are very high also, however. In Fig. 5(d) are reported the values of $r$ obtained from the LZB model and from the experiments in [43]: they almost match. This demonstrates indirectly that our values are realistic values close to the experimental ones. We infer that the dissipation visible on the $\theta(t)$ responses in Figs. 5(a), (b), and (c) may be due mainly to the rebound phases that involve many impacts before the contact corner stabilizes on the base (as experimentally evidenced in [32]), and possibly to sliding during the impacts.

(ii) The fitted value for $e_{n}$ had to be decreased after some impacts for both specimens 1 and 4. Arguments concerning the two-point contact assumption, the line contact effects and the effective width are discussed in [42]; see also [47]. It is noteworthy that such switching process has not been necessary for the bouncing dimer (see [65] and [35]). However, the bouncing dimer has two physical contact points (two sphere/plane contact points), whereas the two-contact-point model is a rough approximation of the line/line contact for the planar block/anvil system. Line/line or even plane/plane effects together with low normal velocities may explain the necessity of the switch in the fitted values for $e_{n}$.

(iii) A detailed analysis of the angular restitution coefficient $r=\frac{\dot{\theta}\left(t^{+}\right)}{\dot{\theta}\left(t^{-}\right)}$is proposed in [63, Sects. 6.1.2, 6.3]. The major conclusion is that $r$ varies very little during the established rocking motions, despite its first value may differ by a small percentage from the other values (see Figs. 43, 44, 45, and 56 in [63]), and that it varies little when $e_{n}$ varies, see Table 9 in [63]. This suggests that a kinematic restitution law with angular velocity restitution might be used, provided that $r$ can be chosen within the admissible domain defined by the kinematic, kinetic, and energetic constraints [11] (see also comment (iv) below). However, an important part of the dynamics like the rebound phases after the first impact, may be missed in many instances of slender blocks. The transient behavior before rocking occurs cannot be predicted by such an impact model (see Sect. 5). We infer that this kinematic law does represent the rocking motion only when there is tangential sticking at impacts and outside impacts, and when the rebound phases vanish (such cases have been experimentally shown, see [32] with $\frac{l}{L}=8$ ). Moreover, such a kinematic law does not permit to predict that a particular motion will occur: it can just be fitted a posteriori. If, anyway, one knows in advance that perfect rocking ${ }^{3}$ with sticking (or almost sticking) contact/impact points is going to occur, then a kinematic law may be preferred because of its simplicity that may be important for calculations.

(iv) In Fig. 5(d) are plotted three angular restitution coefficients: computed and averaged from the LZB simulations with the above fitted parameters $r_{\mathrm{LZB}}$, from the experiments

\footnotetext{
${ }^{3}$ Perfect rocking means that the block rocks without any tangential slip, and with no rebound at the impacting corners.
} 
Fig. 6 Specimen $2, e_{n}=0.99$

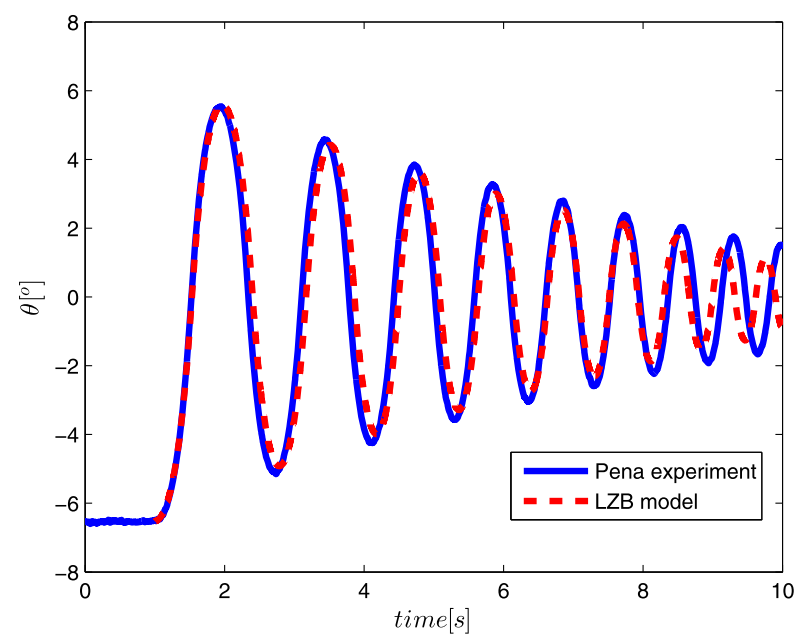

in [43] $r_{\text {exp }}$, and the Housner coefficient obtained from the conservation of angular momentum $r=\frac{L^{2}-2 l^{2}}{2 L^{2}+2 l^{2}}$. The conservation of angular momentum hypothesis usually yields an underestimation of $r$. This is quite visible in Figs. 5(a), (b), and (c) where the Housner value of $r$ yields a much too big dissipation. This is consistent with the results reported in the fourth row of Table 1 in [11].

(v) A very good fitting has been obtained for specimen 2, with $e_{n}=0.999$ in Fig. 5(b). As we shall see in Sects. 4 and 5, the persistent rocking motion and the onset of rocking with base excitation are better fitted with $e_{n}=0.99$. One observes in Fig. 6 that the choice $e_{n}=0.99$ provides a very good fitting for the first oscillations, with a small divergence after $8.5 \mathrm{~s}$. The underlying question is whether the free-rocking experiments can be used to fit the parameters for the base-excited simulations. The relative difference between the two fitted $e_{n}$ is less than $1 \%$. In view of the high sensitivity of the block responses with respect to such parameters, it seems unavoidable that an additional process for parameter identification may be necessary in some instances.

\section{Rocking motion with harmonic base excitation}

Experiments with horizontal base excitation are presented in [43]. We consider here the specimens 1 and 2 in [43]. The base excitation has the form $x_{b}(t)=\mathbf{A} \sin (\omega t)$. The parameters $e_{n}$ and $\mu, \mu_{s}$ in the LZB model are those obtained from the free-rocking fitting process, i.e., $e_{n}=0.97$ for specimen $1, e_{n}=0.99$ for specimen $2, \mu=0.3, \mu_{s}=0.577, l=1 \mathrm{~m}$ and $L=0.23 \mathrm{~m}$ for specimen $1, l=1 \mathrm{~m}$ and $L=0.155 \mathrm{~m}$ for specimen 2 . The results are reported in Fig. 7. It is seen that the LZB model has the tendency to underestimate the peaks magnitudes, however, the frequency of the response is very well predicted. As shown in Fig. 12 in [43] through repeatability tests, the amplitude of the rocking angle $\theta(t)$ may vary from one experiment to the other, which may explain that the LZB model does not predict the same amplitude as in the experimental figures. We may anyway conclude that there is a very good matching between the numerical results and the experimental ones using the free-rocking fitted parameters, taking into account comment (v) of Sect. 3.2.

Remark 3 The DEM method employed for the simulations in [42] seems to provide comparable results to the LZB/complementarity one; see Fig. 7 in [42] that concerns specimen 2 
Fig. $7 \quad \theta(t)$ responses with base excitation

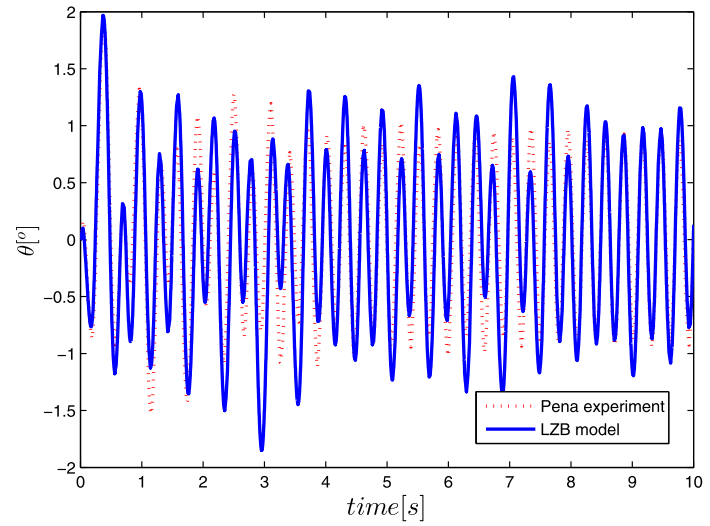

(a) Frequency $\frac{\omega}{2 \pi}=3.3 \mathrm{hz}$, amplitude $\mathbf{A}=6 \mathrm{~mm}$ (specimen 1)

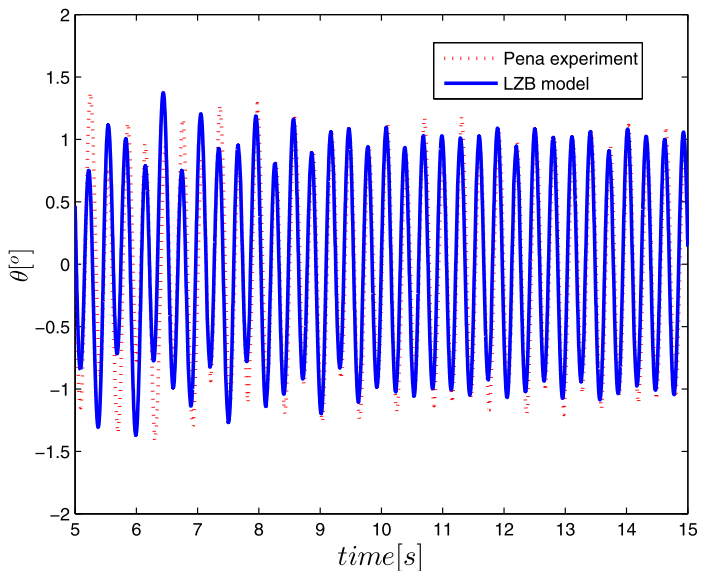

(b) Frequency $\frac{\omega}{2 \pi}=3.3 \mathrm{hz}$, amplitude $\mathbf{A}=8 \mathrm{~mm}$ (specimen 2)

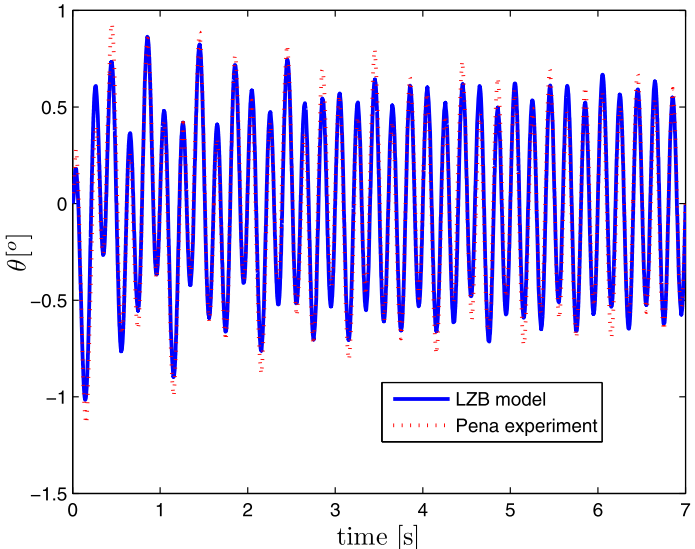

(c) Frequency $\frac{\omega}{2 \pi}=3.3 \mathrm{hz}$, amplitude $\mathbf{A}=5 \mathrm{~mm}$ (specimen 2)

with $\mathbf{A}=5 \mathrm{~mm}, \frac{\omega}{2 \pi}=5 \mathrm{~Hz}$. It is difficult at this stage of the studies to determine which of the two methods is the best one. It is, however, noteworthy that we show in this paper that 
the LZB/complementarity model and event-driven code, is able to correctly predict several types of motions (free-rocking, rocking with horizontal motion, onset of rocking). It is also recalled that the LZB model successfully predicted various motions of different impacting systems like chains of balls [34, 36, 39] and bouncing dimer [65]. It therefore encapsulates the main dynamical effects, which are necessary to well simulate multiple impacts for rateindependent materials, with few parameters that possess a clear physical meaning for both the tangential and the normal dissipation effects.

\section{Onset of rocking with harmonic base excitation}

To start with, the onset of rocking motion in the $(\mathbf{A}, \omega)$ plane is depicted in Fig. 8(a) with the experimental data taken from Table 4 in [43]. They concern specimen 2 with $l=1 \mathrm{~m}$, $L=0.155 \mathrm{~m}, m=228 \mathrm{~kg}$. In all of this section $e_{n}=0.99, \mu=0.3$, and $\mu_{s}=0.577$. A good

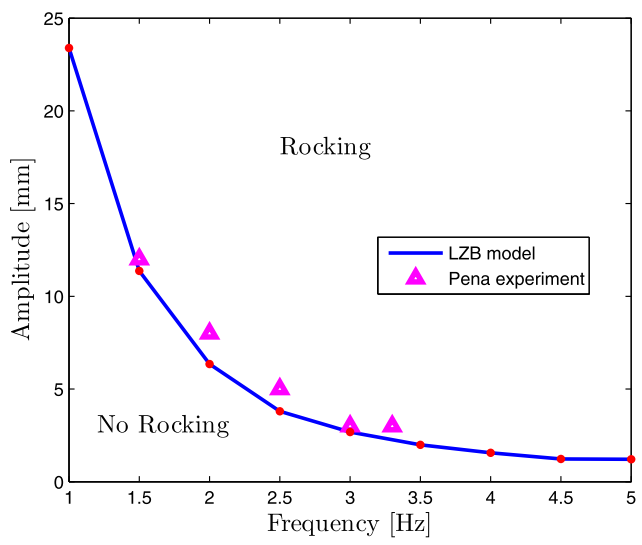

(a) Onset of rocking for specimen 2 .

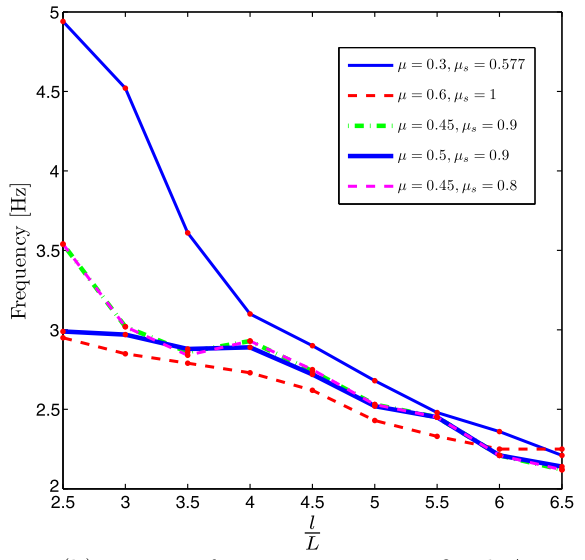

(b) Varying friction parameter, fixed $A$.

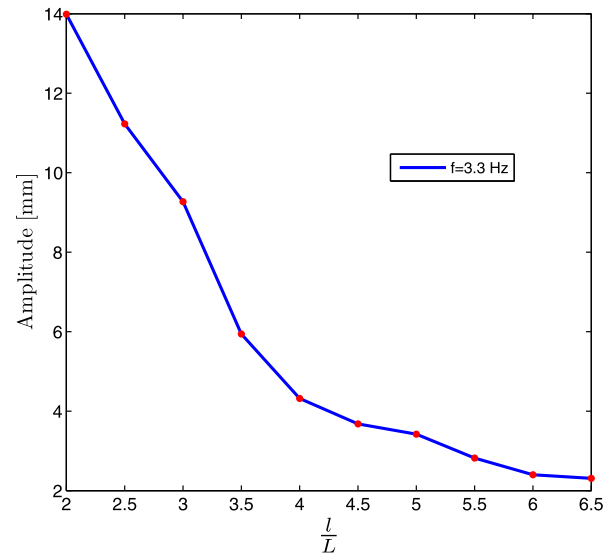

(c) $L=0.155 \mathrm{~m}, \mu=0.3, \mu_{s}=0.577$, fixed $\omega$.

Fig. 8 The onset of rocking 
matching is found between the numerical and the experimental results. ${ }^{4}$ Notice that it is considered that rocking is initiated if the block not only starts to rock on the base, but if this rocking motion is persistent in time (i.e., the mere detachment of one contact point is not sufficient to decide for rocking). The tendency is that rocking starts to occur for large $\mathbf{A}$ when $\omega$ is small, and for small $\mathbf{A}$ when $\omega$ is large. This tendency is also in agreement with the experimental data of Fig. 14 in [57].

Figure 8(b) depicts the onset of rocking for base amplitude $\mathbf{A}=5 \mathrm{~mm}$. The friction parameters are varied. For high enough friction, the onset of rocking occurs almost independently of the friction, but depends mainly on the aspect ratio $\frac{l}{L}$, corroborating previous results [57]. For small enough friction, however, there exists a minimum aspect ratio $\frac{l}{L}$ under which the onset of rocking starts to depend a lot on friction. When the friction is high enough, the contacts statuses are mostly stick, therefore, the value $\mu$ does not count whereas $\mu_{s}$ plays a role. The results for $\frac{l}{L}<2$ are not depicted in Fig. 8(b). Indeed for such values of the aspect ratio and for realistic values of friction, the onset or rocking occurs for very large values of the frequency and one may infer that rocking never occurs in practice.

Let us now consider Fig. 8(c), which depicts the onset of rocking as a function of the aspect ratio $\frac{l}{L}$ and the amplitude $\mathbf{A}$, for a fixed frequency $f=\frac{\omega}{2 \pi}=3.3 \mathrm{~Hz}$. The points on the curve represent the lower limit of the necessary $\mathbf{A}$ for onset of rocking, i.e., rocking occurs for magnitudes just larger. Flat blocks need a large $\mathbf{A}$ to rock, while slender blocks rock for small A. This seems intuitively clear. Figure 9 are depicted the trajectories $\theta(t)$ and the relative tangential velocity $\dot{x}_{\text {rel }}(t)$ at the contact point $A$, for amplitude $\mathbf{A}=5.94 \mathrm{~mm}$ just below the amplitude that triggers rocking and frequency $f=3.3 \mathrm{~Hz}$, and the amplitude such that there is onset of rocking $\mathbf{A}=5.95 \mathrm{~mm}$. These trajectories show that the block motion before rocking settles, is far from an all-stick motion (stick in the tangential direction, and with no impacts), but usually possesses velocity jumps and stick/slip transitions (acceleration jumps). The fact that the complementarity + LZB model is able to predict the onset of rocking after such a complex transient dynamics, with few parameters fitted from the free-rocking experiments, proves that is does encapsulate the main dynamical effects of the block/anvil system.

Finally, Fig. 10 depicts the variation of the minimum value of the product $A f^{2}$ that determines the onset of rocking, as a function of $\frac{l}{L}$. Notice that this quantity is directly related to the maximum acceleration of the base. The values of Fig. 10 are obtained from the average of several values obtained by varying both $A$ and $f$ (see the data in Tables 17 and 18 in [63]). It is clear that the minimum $A f^{2}$ tends to infinity as $\frac{l}{L}$ tends to zero (very flat blocks), while it tends to zero as $\frac{l}{L}$ diverges (very slender blocks). The curve is very regular (and exponential-like) for aspect ratios $\geq 3$. Previous works report a criterion for the onset of rocking without sliding [27, 44, 49], which in fact reduces to a static equilibrium criterion $\mu_{s} \geq \frac{L}{l}$ (see, e.g., Eq. (32) in [27]). The results obtained here do not make the sticking assumption, and some slipping phases are possible both outside and during the impacts, as evidenced in Fig. 9. The maximal acceleration of the base is known to play a significant role in the block dynamics; see, e.g., [26] where this is denoted as PTA (for Peak Table Acceleration) and used to study the so-called engineering demand parameter in purely sliding motions. It is also noteworthy that our results are not based on some model approximations as done sometimes [1], but keep the full nonlinearity of the dynamics in addition to the nonsmooth features.

\footnotetext{
${ }^{4}$ It is important to notice here that the experiments in [43] have been led for a discrete set of amplitude values, for obvious experimental constraints. Thus, the experimentally obtained minimum amplitudes, for a given frequency, are necessarily larger than the numerical ones, which have been computed from a much finer set of amplitude values. This explains the discrepancies between the red dots and the triangles in Fig. 8(a).
} 

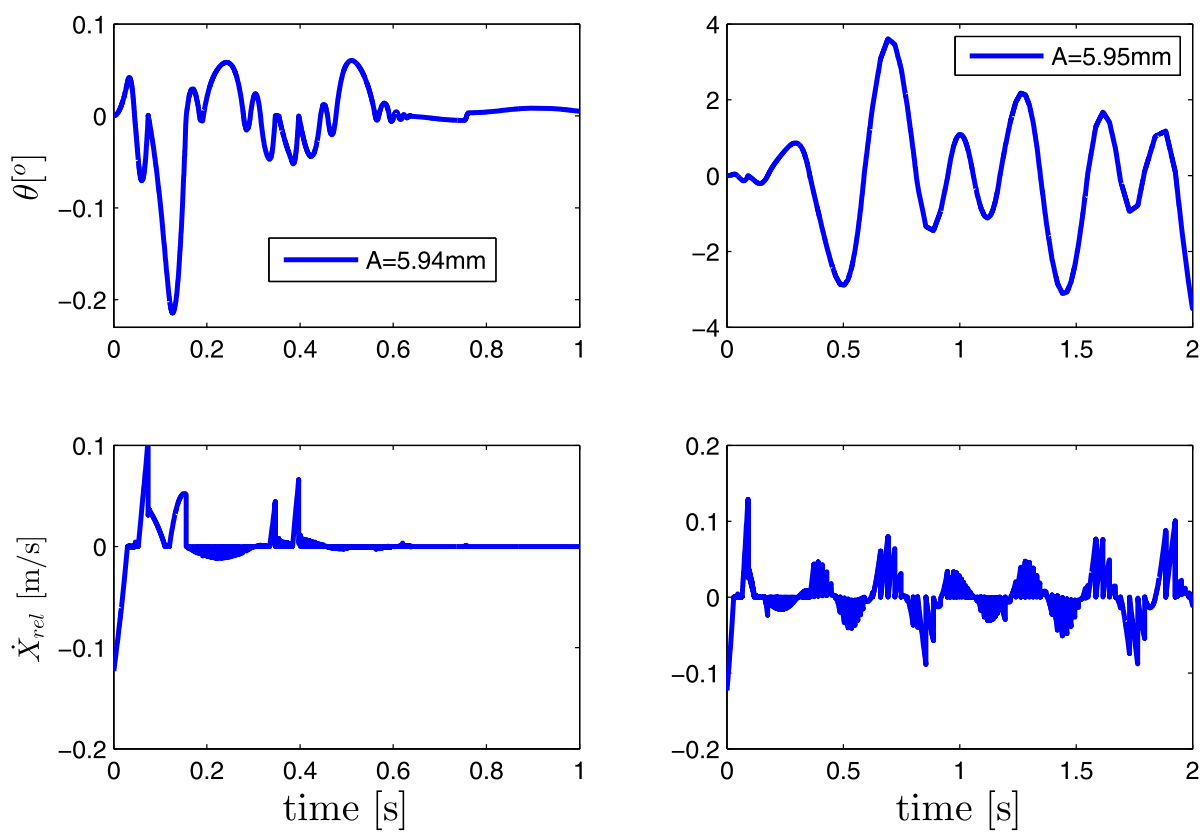

Fig. 9 Responses $\theta(t)$ and relative tangential velocity, $\mu=0.3, \mu_{s}=0.577, f=3.3 \mathrm{~Hz}, \frac{l}{L}=3.5, \mathbf{A}=5.94$ $\mathrm{mm}$ (left column), and $\mathbf{A}=5.95$ (right column)

Fig. $10 A f^{2}$ that triggers rocking

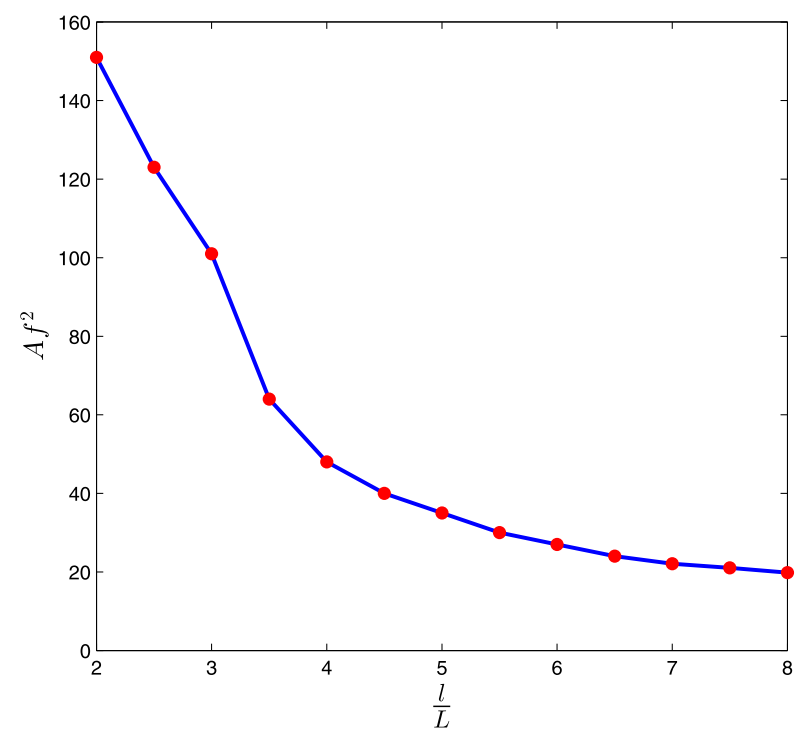

\section{Overturning phenomenon}

In this section, the overturning phenomenon is studied numerically, where the classical harmonic and pulse-based excitations are used. Many studies have been devoted to the over- 
turning phenomenon; see e.g. [1, 3, 14, 17, 26-28, 47, 53, 55, 57]. In particular, several works aim at defining simple enough criteria that may be used to assert if a block is likely to overturn or not. Our goal in this section is rather to prove that the LZB model may be useful to study the overturning because it encapsulates the rich dynamics of the block/ground system. Comparisons with existing numerical studies on the overturning are presented.

\subsection{Harmonic base excitation}

We first consider a horizontal harmonic motion of the base of the above form $x_{b}(t)=$ A $\sin (\omega t)$. It is expected that the overturning phenomenon hardly obeys simple rules, because it is known that the block dynamics with moving base is an extremely sensitive process with respect to initial data and parameters, especially when restitution is high and stick/slip occurs [25]. In all the figures of this section, the curves are numbered starting with the smallest magnitude, or with the smallest frequency. The overturning is the result of an "optimal" exchange of energy between the base and the block, through an increase of the block's oscillation magnitude. This is illustrated in Fig. 11(a), where the $\theta(t)$ response is depicted during $6 \mathrm{~s}$, with frequency $\frac{\omega}{2 \pi}=3.3 \mathrm{~Hz}, e_{n}=0.99, \frac{l}{L}=6$, and $L=0.155 \mathrm{~m}, \mu=0.3, \mu_{s}=0.577$. This figure demonstrates that overturning may occur quickly for $\mathbf{A}=40,55,60,70,30,50 \mathrm{~mm}$, later for $\mathbf{A}=25,35,45,65 \mathrm{~mm}$, and not overturn for $\mathbf{A}=15 \mathrm{~mm}$ before $6 \mathrm{~s}$. In all cases, the block motion before the overturn is quite similar in frequency and amplitude. This indicates that the overturn is the result of a sudden "break" in the base/block relative motion. Figure 11(b) shows that decreasing $e_{n}$, i.e., adding normal dissipation at the impacts, decreases significantly the risk of overturning since all amplitudes $\mathbf{A} \leq 40 \mathrm{~mm}$ yield stable rocking. Figure 12(a) shows that decreasing $\mathbf{A}$ may yield a stable rocking motion after some transient, as may be expected. In Fig. 12(b), the same study is done with varying frequencies and fixed $\mathbf{A}=3 \mathrm{~mm}$. Similar conclusions as for the varying amplitude can be drawn, that there is no monotonic variation of the overturning phenomenon as a function of the base frequency. Discontinuities in the dynamical behavior are common in systems with impact and Coulomb friction. It is expected that more energy dissipation is going to prevent the block from overturning. Dissipation may come from two sources: sliding motions and normal restitution. The friction between the base and the block mainly influences the onset of rocking, for if $\mu=\mu_{s}=0$ the block's corners never detach from the base. However, when rocking has been established the most efficient way to "control" the overturning via energy dissipation is through the normal restitution, as demonstrated in Fig. 11(b). The base excitation is a persistent one. This means that it is difficult to assert firmly whether a motion is really stable or not, because the mechanism of energy transmission between the base and the block is very complex. For instance, we cannot say if the motion with $\mathbf{A}=40 \mathrm{~mm}$ in Fig. 11(b) is stable or not on a long term. This is why studying overturning with simpler base excitations like one-sine period only, may be useful. This is done in the next section.

\subsection{Pulse-type base excitation}

Let us consider that the base has the pulse-type motion (called one-sine type-A pulse in [26, $37,61])$ with an acceleration equal to

$$
\dot{v}_{b}(t)= \begin{cases}a_{p} \sin \left(\omega_{p} t+\psi\right) & \text { if }-\frac{\psi}{\omega_{p}} \leq t \leq \frac{2 \pi-\psi}{\omega_{p}} \\ 0 & \text { otherwise }\end{cases}
$$


Fig. 11 The overturning with varying $\mathbf{A}, f=3.3 \mathrm{~Hz}$

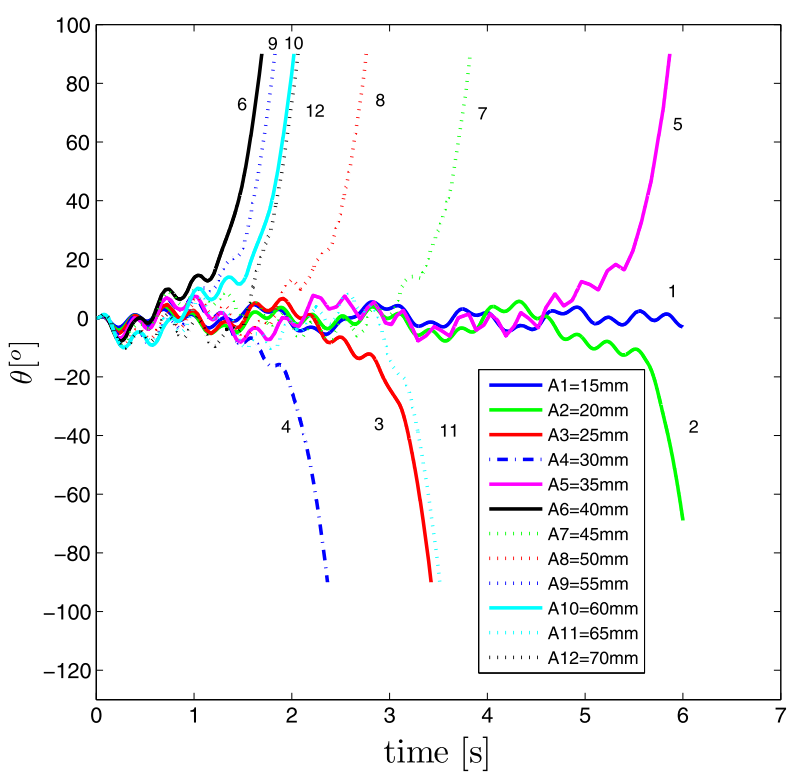

(a) Overturning amplitudes, $e_{\mathrm{n}}=0.99$.

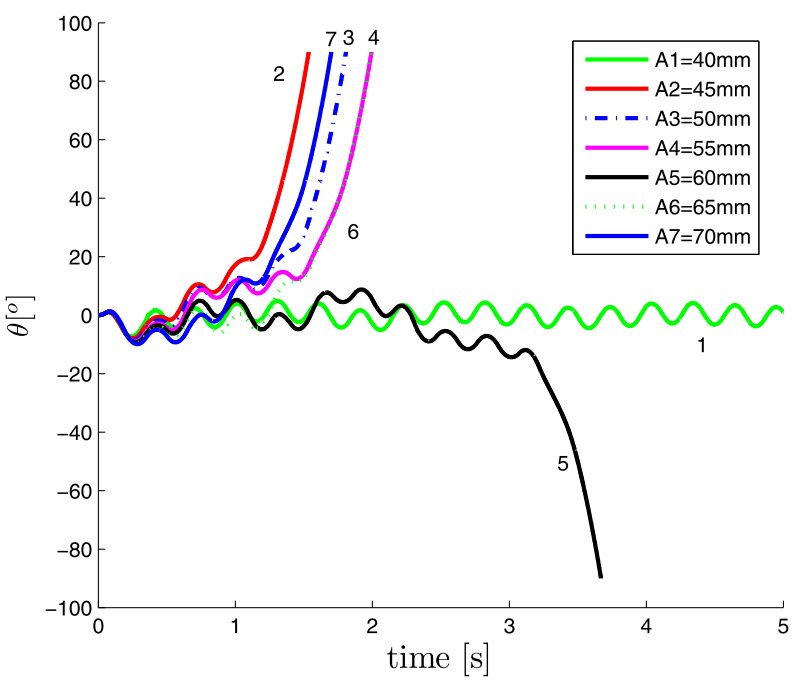

(b) Overturning amplitudes, $e_{\mathrm{n}}=0.8$.

with $\psi=\arcsin \left(\frac{\alpha g}{a_{p}}\right), \alpha=\arctan \left(\frac{L}{l}\right)$. The advantage of considering such a base excitation is that it allows one to clearly separate the motions that overturn and those that do not overturn. Indeed, once the base is at rest, the block may only lose energy. If it has not overturned before it starts to lose its energy, it will never overturn. This has been used in [26, 61], where one can find numerical results about the safe and unsafe areas depending on the amplitude and frequency of the base excitation (see, for instance, Fig. 6 in [26]). The results are reported in Fig. 13(a). The parameters are chosen as in [37, Fig. 5], which is reproduced in Fig. 13(b), i.e., $l=1.555, L=0.3971, e_{n}=0.9$, so that $p \triangleq \sqrt{\frac{3 g}{4 R}}=2.14$ and $\alpha=\arctan \left(\frac{L}{l}\right)=0.25$. 
Fig. 12 The overturning with varying frequency and amplitude

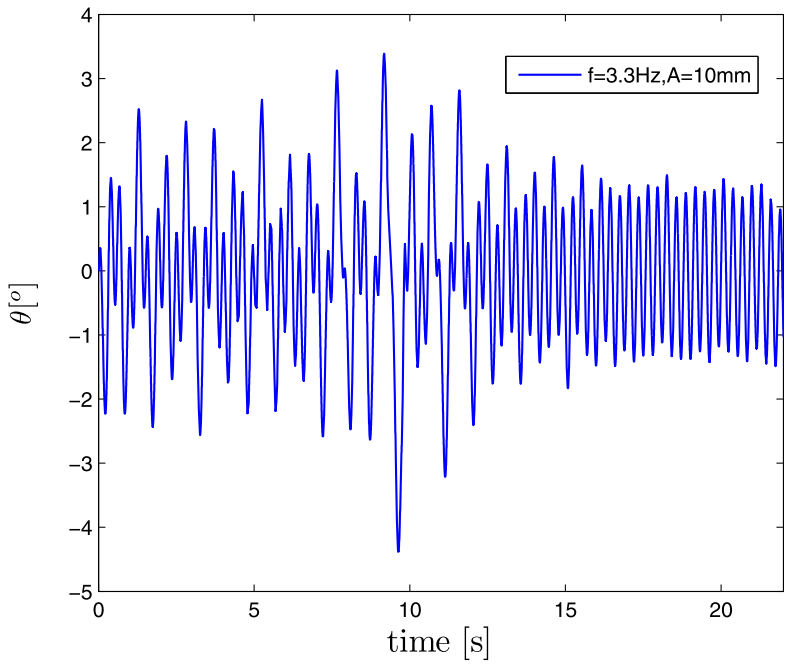

(a) Non-overturning amplitude.

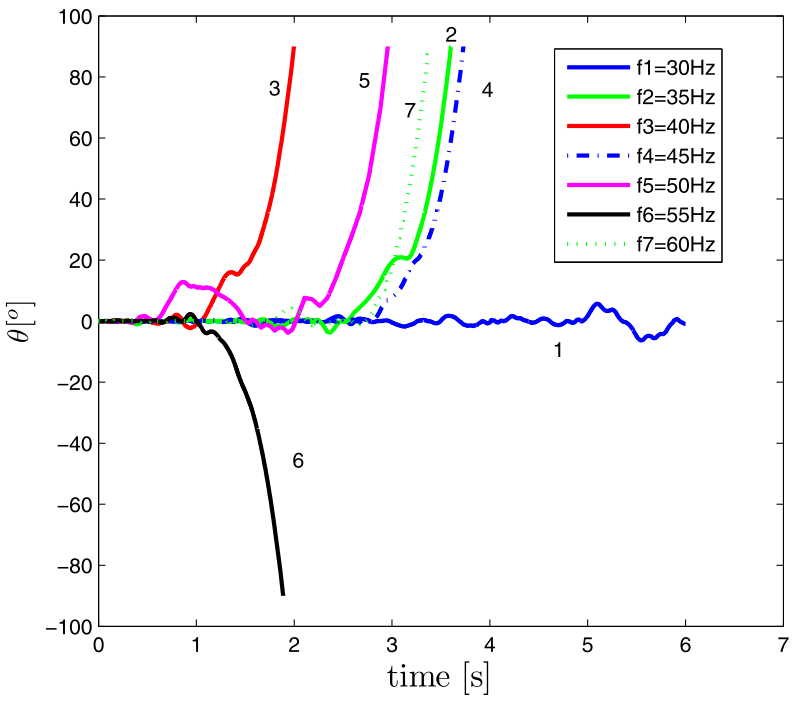

(b) Overturning with varying $\omega, \mathbf{A}=3 \mathrm{~mm}$.

- In Fig. 13(a) are depicted various areas in the $\left(\frac{a_{p}}{\alpha g}, \frac{\omega_{p}}{p}\right)$ plane. Qualitatively, we recover the same shapes as in [26, Fig. 6] or [37, Figs. 5, 8, 9]: a big area within which overturning occurs with no impact, and a smaller "tongue shaped" area where overturning occurs after one or several impacts. In more detail:

$-\mu=0.3, \mu_{s}=0.577$ : overturn with no impact in $A G H$, with one or two impacts in $A F E C B A$.

$-\mu=0.3, \mu_{s}=0.8$ : overturn with no impact in $A G H$, with one or two impacts in $A F I D C B A$.

$-\mu=0.5, \mu_{s}=0.8$ : overturn with no impact in $A G J K$, with one or two impacts in AFIDCBA. 


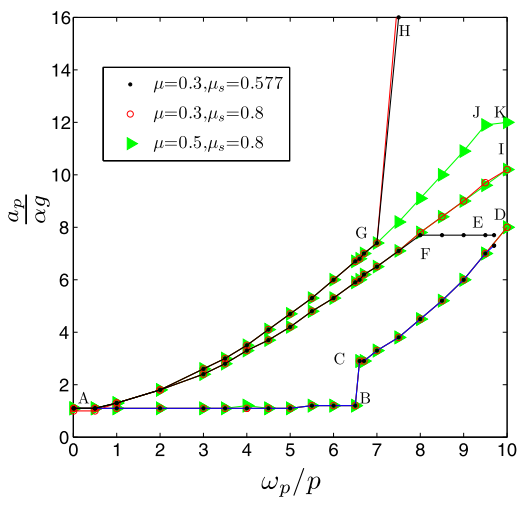

(a) Results with the LZB/complementarity model.

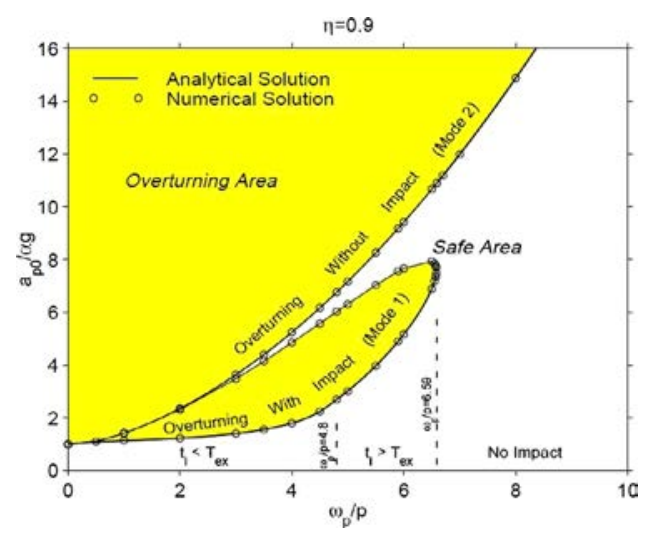

(b) Fig. 5 in [37].

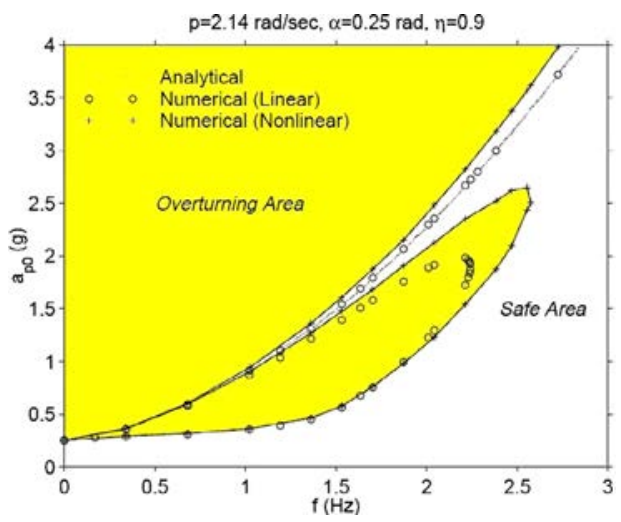

(c) Fig. 8 in [37].

Fig. 13 Overturning safe and unsafe areas

The tongue-shaped areas have been reported numerically and with some experimental validations on overturning in [17, Figs. $6,7,8]$. They have been computed numerically in various papers [14, 26, 28, 55].

- The transitions between the various areas in Fig. 13(a) are illustrated in Figs. 67, 68, 69 in [63]. In particular, the number of impacts before the overturn event may be seen on the $\theta(t)$ curves (a), (b), (c) of these figures. There are, however, some discrepancies with respect to the results in [26, 37, 61]; see also [27]:

- Consider the case $\mu=0.5, \mu_{s}=0.8$. The overturn in the area above the line $A B$ and below the line $A F$, occurs after two impacts (which is new compared to [26, Fig. 6] and to Fig. 13(b), which indicate only one impact). There is a discontinuity between points $B$ and $C$, because the overturning in the area above $C D$ and below the next curve $A F I$ occurs with one impact only. The result is that the LZB model considers that overturn may occur for much higher frequencies than the linear model used in [37] to establish Fig. 5 in that paper. Notice that the break at $B C$ corresponds to the largest frequency of the one-impact overturn area of Fig. 13(b). 
- The overturning area with one or two impacts, is much larger than the one-impact overturning area in [26, Fig. 6] or [27, Fig. 11]. It is noteworthy that Fig. 11 in [27] and Figs. 8 and 9 in [37] already show a big discrepancy between the two models used in these papers (called therein the linear and the nonlinear models), as illustrated in Fig. 13(c) (the scales are not the same but are presented in [37] so that the two figures can still be compared). Our simulations are led with the full smooth nonlinearities, and with in addition the stick/slip modes. This indicates that simplifying too much the dynamical equations and neglecting the smooth nonlinearities in (2) as well as Coulomb's friction modes outside and during the impacts, may result in an overestimation of the safe area of no overturn: as long as new dynamical effects are added to the model, the safe area decreases as is visible starting from Fig. 13(b) then (c) then (a). As shown in [37, Fig. 14], the discrepancy between the results provided by the various models also depends on the type of excitation that is applied on the base. We may infer that much more work is needed in the future to determine safe areas as a function of all parameters: aspect ratio, contact parameters, base excitation.

- As expected, the safe area increases when the friction decreases that indicates that more slip implies less overturn. In the frictionless limit, there is no overturn since the block keeps slipping on the base.

- Figs. 14(a), (d), (g) correspond to a point on the line $A B$ in Fig. 13, Figs. 14(b), (e), and (h) correspond to a point on the line $C D$, Figs. 14(c), (f), and (i) correspond to a point on the line $A F$. From Figs. 14(g), (h), and (i), one sees that the overturning is almost always occurring after a phase of slip, followed by a sticking phase at the point of contact (notice that the vertical scales in Figs. 14(g), (h), and (i) and in Figs. 14(d), (e), and (f) are quite different in magnitude).

The overturning phenomenon is certainly the most complex phenomenon that may occur in the block/ground system. Our numerical results mainly aim at showing that the LZB model with friction, coupled to the complementarity system in (2) outside the impacts, may improve our knowledge about overturning in planar blocks. It is to be considered as a preliminary work because on the first hand three-dimensional effects are likely to play a significant role in most of the experiments with strong base excitation, on the second hand, real earthquakes excitations are more complex than those considered here.

\section{Conclusions}

This paper focusses on the experimental validation of the multiple impact law with Coulomb friction introduced in [33-36, 65] on the planar block/anvil system. This impact law is based on the Darboux-Keller assumptions, and is a rigid body model incorporating local flexibility effects at the contact points, with few parameters per contact (restitution and friction coefficients). Detailed comparisons between the numerical results obtained with an event-driven method and the experimental data found in $[42,43]$ are made. It is shown that the simple, free-rocking experiments, may be used to fit the parameters of the impact law for simulating more complex dynamics with base excitation. Compared to other models widely used in the earthquake engineering literature, our model incorporates Coulomb friction and allows for bounces at the contact corner, a fact experimentally evidenced elsewhere [32]. In particular, this allows to simulate the transient behaviour that occurs before the onset of rocking, which is shown to consist of stick/slip and impact events that can hardly be neglected. It also has the advantage of using few parameters. This work is a preliminary validation of a recently introduced multiple impact law with Coulomb friction, on a two-body, two-dimensional system. Future works should concern in priority: 
(a)

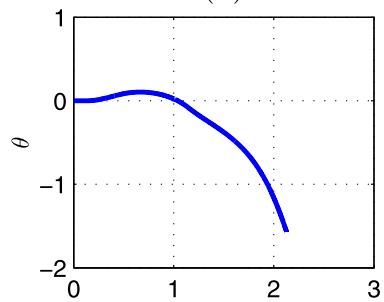

$(d)$

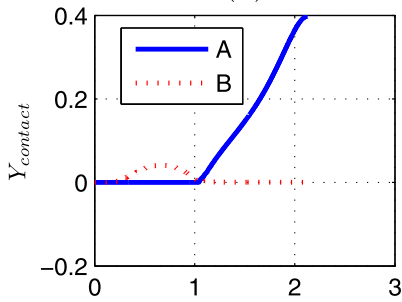

(g)

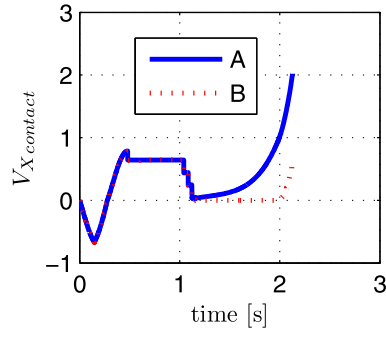

(b)

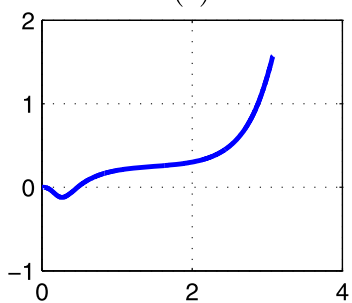

(e)

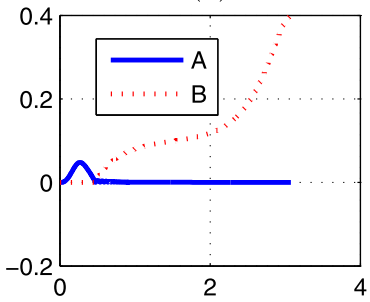

(h)

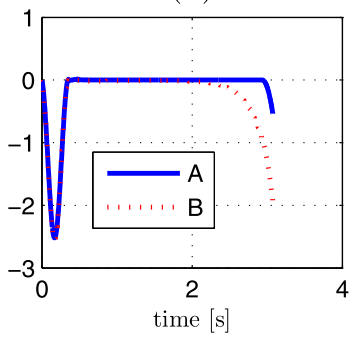

(c)

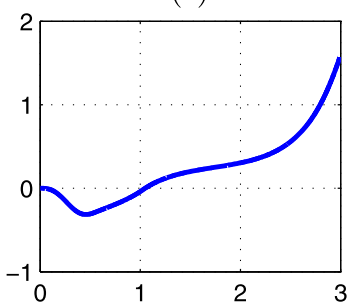

(f)

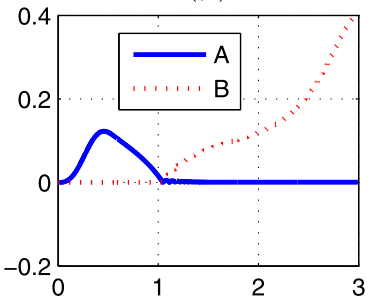

(i)

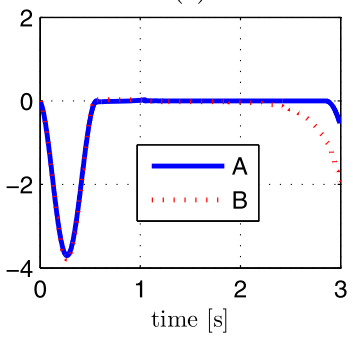

Fig. 14 (a), (b), (c): $\theta(t),(\mathbf{d}),(\mathbf{e}),(\mathbf{f})$ vertical positions, and (g), (h), (i) relative horizontal velocities of the contact points

- the analysis of the three-dimensional block/anvil system;

- incorporating line/line or plane/plane contact models, lumped flexibilities in the structure, asymmetry in the block geometry;

- more complex base excitations, like real earthquake and random excitations;

- stacked blocks.

Acknowledgements H. Zhang's work performed while at INRIA, BIPOP project-team, ZIRST Montbonnot, 655 avenue de l'Europe, 38334 Saint Ismier, France. Funded by China Scholarship Council No. 2009601276 and by ANR project Multiple Impact ANR-08-BLAN-0321-01.

The authors are very grateful to Dr. Fernando Pena (Instituto de Ingenieria, UNAM Mexico) for providing them with detailed data from the experiments in [43]. These data have been valuable for achieving this work. This work was performed with the support of the NSFC/ANR project Multiple Impact, ANR-08-BLAN0321-01, and with support of the NSFC key project (Grant No. 11132001).

\section{References}

1. Al Abadi, H., Lam, N., Gad, E.: A simple displacement-based model for predicting seismically induced overturning. J. Earthq. Eng. 10(6), 775-814 (2006) 
2. Acary, V., Brogliato, B.: Numerical Simulation for Nonsmooth Dynamical Systems. Lecture Notes in Applied and Computational Mechanics, vol. 35. Springer, Heidelberg (2008)

3. Acikgoz, S., DeJong, M.: The interaction of elasticity and rocking in flexible structures allowed to uplift. Earthq. Eng. Struct. Dyn. 41(15), 2177-2194 (2012). doi:10.1002/eqe.2181

4. Andreaus, U., Casini, P.: On the rocking-uplifting motion of a rigid block in free and forced motion: influence of sliding and bouncing. Acta Mech. 138, 219-241 (1999)

5. Andreaus, U., Casini, P.: Dynamics of three-block assemblies with unilateral deformable contacts. Part 1: contact modelling. Earthq. Eng. Struct. Dyn. 28, 1621-1636 (1999)

6. Antonyuk, S., Heinrich, S., Tomas, J., Deen, N.G., van Buijtenen, M.S., Kuipers, J.A.M.: Energy absorption during compression and impact of dry elastic-plastic spherical granules. Granul. Matter 12(1), $15-47$ (2010)

7. Aslam, M., Godden, W.G., Scalise, D.T.: Earthquake rocking response of rigid bodies. J. Struct. Eng. 106(2), 377-392 (1980)

8. Bernasconi, D.J., Fandrich, M.E., Hogue, C.: Experimental 3-D impact analysis. Multibody Syst. Dyn. 1(4), 361-379 (1997)

9. Bowling, A., Montrallo Flickinger, D., Harmeyer, S.: Energetically consistent simulation of simultaneous impacts and contacts in multibody systems with friction. Multibody Syst. Dyn. 22(1), $27-45$ (2009)

10. Brogliato, B.: Nonsmooth Mechanics, 2nd edn. Springer, London (1999)

11. Brogliato, B., Zhang, H., Liu, C.: Analysis of a generalized kinematic impact law for multibodymulticontact systems, with application to the planar rocking block and chains of balls. Multibody Syst. Dyn. 27, 351-382 (2012)

12. Crook, A.W.: A study of some impacts between metal bodies by a piezo-electric method. Proc. R. Soc. Lond. Ser. A, Math. Phys. Sci. 212(1110), 377-390 (1952). 7

13. Cross, R.: The bounce of a ball. Am. J. Phys. 67(3), 222-227 (1999)

14. Dimitrakopoulos, E.G., DeJong, M.J.: Revisiting the rocking block: closed-form solutions and similarity laws. Proc. R. Soc. A (2012). doi:10.1098/rspa.2012.0026

15. Djerassi, S.: Collision with friction. Part A: Newton's hypothesis. Multibody Syst. Dyn. 29, 37-54 (2009)

16. ElGawady, M.A., Ma, Q., Butterworth, J.W., Ingham, J.: Effects of interface material on the performance of free rocking blocks. Earthq. Eng. Struct. Dyn. 40(4), 375-392 (2011)

17. Fielder, W.T., Virgin, L.N., Plaut, R.H.: Experiments and simulation of overturning of an asymmetric rocking block on an oscillating foundation. Eur. J. Mech. A, Solids 16(5), 905-923 (1997)

18. Montrallo Flickinger, D., Bowling, A.: Simultaneous oblique impacts and contacts in multibody systems with friction. Multibody Syst. Dyn. 23(3), 249-261 (2010)

19. Flores, P., Ambrosio, J.: On the contact detection for contact-impact analysis in multibody systems. Multibody Syst. Dyn. 24(1), 103-122 (2010)

20. Glocker, Ch.: Concepts for modeling impacts without friction. Acta Mech. 168, 1-19 (2004)

21. Goldsmith, W.: Impact. The Theory and Physical Behaviour of Colliding Solids. Edward Arnold Publishers, London (1960)

22. Housner, G.W.: The behaviour of inverted pendulum structures during earthquakes. Bull. Seismol. Soc. Am. 53(2), 403-417 (1963)

23. Imre, B., Rabsamen, S., Springman, S.M.: A coefficient of restitution of rock materials. Comput. Geosci. 37, 339-350 (2008)

24. Ishiyama, Y.: Motions of rigid bodies and criteria for overturning by earthquake excitations. Earthq. Eng. Struct. Dyn. 10, 635-650 (1982)

25. Jeong, M.Y., Suzuki, K., Yim, S.C.S.: Chaotic rocking behavior of freestanding objects with sliding motion. J. Sound Vib. 262, 1091-1112 (2003)

26. Konstantinidis, D., Makris, N.: Experimental and analytical studies on the responses of 1/4-scale models of freestanding laboratory equipment subjected to strong earthquake shaking. Bull. Earthq. Eng. 8, 14571477 (2010)

27. Kounadis, A.N.: On the overturning instability of a rectangular rigid block under ground excitation. Open. Mech. J. 4, 43-57 (2010)

28. Kounadis, A.N., Papadopoulos, G.J., Cotsovos, D.M.: Overturning instability of a two-rigid block system under a ground excitation. Z. Angew. Math. Mech. (2012). doi:10.1002/zamm.201100095

29. Lankarani, H.M., Pereira, M.F.O.S.: Treatment of impact with friction in planar multibody mechanical systems. Multibody Syst. Dyn. 6(3), 203-227 (2001)

30. Leine, R.I., Brogliato, B., Nijmeijer, H.: Periodic motion and bifurcations induced by the Painlevé paradox. Eur. J. Mech. A, Solids 21, 869-896 (2002)

31. Leine, R., van de Wouw, N.: Stability properties of equilibrium sets of non-linear mechanical systems with dry friction and impact. Nonlinear Dyn. 51, 551-583 (2008) 
32. Lipscombe, P.R., Pellegrino, S.: Free rocking of prismatic blocks. J. Eng. Mech. 119(7), 1387-1410 (1993)

33. Liu, C., Zhao, Z., Brogliato, B.: Frictionless multiple impacts in multibody systems. Part I. Theoretical framework. Proc. R. Soc. A, Math. Phys. Eng. Sci. 464(2100), 3193-3211 (2008)

34. Liu, C., Zhao, Z., Brogliato, B.: Energy dissipation and dispersion effects in a granular media. Phys. Rev. E 78(3), 031307 (2008)

35. Liu, C., Zhao, Z., Brogliato, B.: Variable structure dynamics in a bouncing dimer. INRIA Research Report 6718 (2008). http://hal.inria.fr/inria-00337482/fr/

36. Liu, C., Zhao, Z., Brogliato, B.: Frictionless multiple impacts in multibody systems. Part II. Numerical algorithm and simulation results. Proc. R. Soc. A, Math. Phys. Eng. Sci. 465(2101), 1-23 (2009)

37. Makris, N., Zhang, J.: Rocking response and overturning of anchored equipment under seismic excitations. PEER Report 1999/06, Pacific Earthquake Engineering Research Center, College of Engineering, University of California, Berkeley (1999). Available at http://nisee.berkeley.edu/elibrary/Text/1200242

38. Modarres Najafabadi, S.A., Kovecses, J., Angeles, J.: Impacts in multibody systems: modeling and experiments. Multibody Syst. Dyn. 20(2), 163-176 (2008)

39. Nguyen, N.S., Brogliato, B.: Shock dynamics in granular chains: numerical simulations and comparisons with experimental results. Granul. Matter 14(3), 341-362 (2012)

40. Nguyen, N.S., Brogliato, B.: Shock dynamics in granular chains: numerical simulations and comparison with experimental tests. INRIA Research Report RR-7636 (2011) http://hal.inria.fr/inria-00597468

41. Palmeri, A., Makris, N.: Response analysis of rigid structures rocking on viscoelastic foundation. Earthq. Eng. Struct. Dyn. 37, 1039-1063 (2008)

42. Pena, F., Prieto, F., Lourenço, P.B., Campos Costa, A., Lemos, J.V.: On the dynamics of rocking motion of single rigid-block structures. Earthq. Eng. Struct. Dyn. 36, 2383-2399 (2007)

43. Pena, F., Lourenço, P.B., Campos-Costa, A.: Experimental dynamic behavior of free-standing multiblock structures under seismic loadings. J. Earthq. Eng. 12, 953-979 (2008)

44. Pompei, A., Scalia, A., Sumbatyan, M.A.: Dynamics of rigid block due to horizontal ground motion. J. Eng. Mech. 124(7), 713-717 (1998)

45. Priestley, M.J.N., Evenson, R.J., Carr, A.J.: Seismic response analysis of structures free to rock on their foundations. Bull. N. Z. Soc. Earthq. Eng. 11(3), 141-150 (1978)

46. Prieto, F., Lourenço, P.B.: On the rocking behavior of rigid objects. Meccanica 40, 121-133 (2005)

47. Purvance, M.D., Anooshehpoor, A., Brune, J.N.: Freestanding block overturning fragilities: numerical simulation and experimental validation. Earthq. Eng. Struct. Dyn. 37, 791-808 (2008)

48. Rodriguez, A., Bowling, A.: Solution to indeterminate multipoint impact with frictional contact using constraints. Multibody Syst. Dyn. (2012). doi:10.1007/s11044-012-9307-X

49. Shenton, H.W.: Criteria for initiation of slide, rock, and slide-rock rigid-body modes. J. Eng. Mech. 122(7), 690-693 (1996)

50. Spanos, P.D., Koh, A.S.: Rocking of rigid blocks during harmonic shaking. J. Eng. Mech. 110(11), $1627-1642(1984)$

51. Stevens, A.B., Hrenya, C.M.: Comparison of soft-sphere models to measurements of collision properties during normal impacts. Powder Technol. 154, 99-109 (2005)

52. Stronge, W.J.: Impact Mechanics. Cambridge University Press, Cambridge (2000)

53. Taniguchi, T.: Non-linear response analyses of rectangular rigid bodies subjected to horizontal and vertical ground motion. Earthq. Eng. Struct. Dyn. 31, 1481-1500 (2002)

54. Tso, W.K., Wong, C.M.: Steady state rocking response of rigid blocks. Part 1: Analysis. Part 2: Experiment. Earthq. Eng. Struct. Dyn. 18(1), 89-120 (1989)

55. Vassiliou, M.F., Makris, N.: Analysis of the rocking response of rigid blocks standing free on a seismically isolated base. Earthq. Eng. Struct. Dyn. 41(2), 177-196 (2012)

56. Weir, G., Tallon, S.: The coefficient of restitution for normal incident, low velocity particle impacts. Chem. Eng. Sci. 60, 3637-3647 (2005)

57. Winkler, T., Meguro, K., Yamazaki, F.: Response of rigid body assemblies to dynamic excitation. Earthq. Eng. Struct. Dyn. 24, 1389-1408 (1995)

58. Wu, C.Y., li, L.Y., Thorton, C.: Energy dissipation during normal impact of elastic and elastic-plastic spheres. Int. J. Impact Eng. 32, 593-604 (2005)

59. Yilmaz, C., Gharib, M., Hurmuzlu, Y.: Solving frictionless rocking block problem with multiple impacts. Proc. R. Soc. A, Math. Phys. Eng. Sci. 465, 3323-3339 (2009)

60. Yim, C.S., Chopra, A.K., Penzien, J.: Rocking response of rigid blocks to earthquakes. Earthq. Eng. Struct. Dyn. 8(6), 565-587 (1980)

61. Zhang, J., Makris, N.: Rocking response of anchored blocks under pulse-type motions. J. Eng. Mech. 127(5), 411-529 (2001)

62. Zhang, J., Makris, N.: Rocking response of free-standing blocks under cycloidal pulses. J. Eng. Mech. 127(5), 473-483 (2001) 
63. Zhang, H., Brogliato, B.: The planar rocking block: analysis of kinematic restitution laws, and a new rigid-body impact model with friction. INRIA Research Report RR-7580 (2011). http://hal. inria.fr/inria-00579231/en/

64. Zhang, H., Brogliato, B., Liu, C.: Study of the planar rocking-block dynamics without and with friction: critical kinetic angles. J. Comput. Nonlinear Dyn. 8(2), 021002 (2013) (11 pp.)

65. Zhen, Z., Liu, C., Brogliato, B.: Planar dynamics of a rigid body system with frictional impacts. II. Qualitative analysis and numerical simulations. Proc. R. Soc. A, Math. Phys. Eng. Sci. 465(2107), 22672292 (2009)

66. Zhen, Z., Liu, C.: The analysis and simulation for three-dimensional impact with friction. Multibody Syst. Dyn. 18(4), 511-530 (2007)

67. Zulli, D., Contento, A., Di Egidio, D.: 3D model of rigid block with a rectangular base subject to pulsetype excitation. Int. J. Non-Linear Mech. (2012). doi:10.1016/j.ijnonlinmec.2011.11.004 